Lung Disease amongst others. It is, however, counterproductive to suggest that asthma needs to be defined with precision in some absolute sense. This is not possible either for epidemiological or for clinical studies, and is not likely to be possible until after the causes of asthma are more fully understood. What can be done is to standardise methods of diagnosis between studies and between researchers. This is an easier task.

On the interpretation of airway hyperreactivity, I have never held the view that this was the same as "asthma." I I do, however, believe that it is a useful objective marker for the condition. Much is now being written on the imperfect association between asthma and airway hyperreactivity but caution is advisable in interpreting this, as there was in earlier times when airway hyperreactivity was thought by some to be almost synonymous with the condition. Firstly, there is the problem of defining asthma, which Dr Gregg mentions as a problem in epidemiological studies but not, evidently, in comparing "asthma" and airway hyperreactivity. Secondly, there is the inevitable discrepancy between two measures neither of which is perfectly reproducible. None of the studies that I know of in this area have addressed this problem.

Although I tend to believe, like Dr Gregg, that there has been an increase in the prevalence and severity of asthma, I hardly think that the rise is "undoubted"; and I am not sure that an epidemiological programme would be wise to make its major interest an explanation of this increase, as the data that would be required to support any such explanation have largely disappeared. Differences between contemporary populations are much easier to study.

As to the future, we will probably have to remain in disagreement until time tells whose assessment is more accurate. Dr Gregg's view that past failures must predict further failure seems unduly pessimistic. On the other hand, it may be an inevitable cultural prejudice that an epidemiologist sees more hope in understanding the epidemiological data than in disentangling the apparently limitless complexities tilat face the pathophysiologists. I remain relatively sure, none the less, that the pathophysiology will be easier to understand when the cause of the disease is known.

PETER BURNEY

Department of Community Medicine United Medical and Dental Schools of Guy's and St Thomas's Hospitals St Thomas's Campus London SE1 $7 E H$

1 PGJ Burney, JR Britton, S Chinn, et al. Descriptive epidemiology of bronchial reactivity in an adult population: results from a community study. Thorax 1987;42:38-44.

\section{Reproducibility of walking test results in chronic obstructive airways disease}

After we had read the interesting paper by Dr AJ Knox and others (May 1988;43:388-92) we found that some questions were unanswered.

In the study of reproducibility over three consecutive days the authors did not mention the time schedule of the test procedures: only the standardisation criteria for medication before the first walk of each study day were reported. They found a decrease in mean visual analogue scale scores with day and an increase in walking distance both with day and with walk number. They did not report the visual analogue scale values at rest before each walk; possibly a change in breathlessness at rest could have influenced the results.

Furthermore, the authors studied the reproducibility over four consecutive weeks. We note the considerable difference in walking distance between study groups 1 and 2, despite the same spirometric entry criteria, and the opposite changes between groups 1 and 2 when only the first three walk tests are considered. They did not say whether daily activities during these four weeks were standardised; exercise training by the patient could perhaps have influenced the results. Moreover, the time schedule and use of bronchodilators were not mentioned.

R MOSTERT
P SWERTS
EFM WOUTERS
Asthma Centre and Clinic for Chronic Respiratory
Diseases, Horn;
Institution for Rehabilitation Research, Hoensbroek;
Department of Pulmonary Diseases, University Hospital,
Maastricht, The Netherlands

AUTHORS' REPLY We thank Dr Mostert and colleagues for their interest in our paper. With regard to the time schedule, the first walk on each day was always performed at the same time of day with one hour between each walk. Bronchodilators were not allowed between walks on any day.

With regard to visual analogue scale scores, we did not determine these before walks. It is conceivable that resting breathlessness may also have improved with repeated testing. This would be of interest.

Dr Mostert and colleagues point out that there was a difference in walking distance between our two study groups at the start of our study despite the similar spirometric values. There were also slight differences in spirometric values between the two groups (study 1: $F_{E V} 0.71$, forced vital capacity $1.7 \mathrm{l}$; study 2 FEV, $0.8 \mathrm{l}$, FVC $2.0 \mathrm{l}$ ) and these might account for the difference in walking distance. Nevertheless, it would not be surprising if two groups with similar spirometric values did have different walking distances as both our work and the work of others suggests that spirometry is a poor predictor of exercise performance.

Dr Mostert and colleagues also point out that there was a small decrease in walking distance between walks 2 and 3 in our second study. The trend over the 12 walks was upwards in the study, and the small difference between this pair of walks is most likely to reflect "noise." Our study 1 and the studies by McGavin et al, Swinburn et al, Butland et al, and Mungall and Hainsworth, which we quoted in our paper, have all shown increases over three walks.

With regard to the standardisation of daily activities, subjects were asked merely to continue their normal daily activities. While there is no way of ensuring that patients do not take additional exercise, the same is true in clinical practice when walking tests are used to assess treatment benefit. The message of our study is that improvement in walking distance occurs with repeated testing. While this improvement could be attributed to either a learning or an 
exercise training effect, it is important that it is recognised and not falsely attributed to treatment benefit.

ALAN J KNOX

JOHN FJ MORRISON

MARTIN F MUERS

Respiratory Medicine Unit

City Hospital

Nottingham NG5 IPB

Killingbeck Hospital

Leeds LS14 6UQ

\section{Post-pneumonectomy pulmonary oedema}

In the article by Dr L Verheijen-Breemhaar and others (April 1988;43:323-6) I felt that not enough information was given to put the figures into perspective.

What is the incidence of pulmonary oedema after any operation in the general population and, more specifically, after lobectomy in what must be a matched group? There was no mention of anaesthetic technique and this undoubtedly has changed between the years of 1975 and 1988, with the introduction of new induction agents, muscle relaxants, and opiates, all capable of influencing recovery. Epidural anaesthesia with both local anaesthetics and opiates and the use of opiate infusions have meant that a return of pain at the end of surgery, causing an increase in venous return, tachycardia, and increased cardiac output, all capable of precipitating pulmonary oedema, is no longer de rigeur.

The reasons given for pulmonary oedema by the authors should cause problems only immediately after operation and I would be loth to attribute the event on day 7 to such a cause.

Poor conduct of anaesthesia will undoubtedly precipitate pulmonary oedema in these patients - that is, poor analgesia, poor reversal, undue sedation and inability to sit up, and excessive transfusion. Having briefly reviewed 47 consecutive pneumonectomies in this hospital and found no evidence of pulmonary oedema, I consider that it is not an integral part of the postoperative course if attention is paid to anaesthetic detail. Thus I would have appreciated more information on this point.

MDD BELL Leeds General Infirmary Leeds LS9 7 TF

AUTHORS' REPLY In reply to Dr Bell's comments we would like to make the following remarks. Our patients are operated on in a modern cardiopulmonary surgical unit. Anaesthesia techniques do not differ significantly from those generally used. During the 1975-84 period pulmonectomy or lobectomy was performed in 502 patients. In only one patient, who was suffering from chronic cryptogenic alveolitis, pulmonary oedema occurred. Extensive data on the start of symptoms were not presented in the article. In all patients symptoms started within $\mathbf{4 8}$ hours of thoracotomy. In one patient, who underwent a second thoracotomy within 24 hours because of severe postoperative haemorrhage, dyspnoea started on the first postoperative day. Because symptoms progressed very slowly it was one week after pneumonectomy before artificial ventilation had to be instituted. We are convinced that postpneumonectomy pulmonary oedema as described by us and by others is a real entity. In our opinion the data as presented by Dr Bell do not exclude that such a complication $\overrightarrow{\vec{s}}$ may occur. We fully agree, however, that attention to anaesthetic detail, including perioperative and postoperative fluid balance, is paramount in preventing postpneumonec- $\frac{\bar{O}}{\bar{N}}$ tomy oedema.

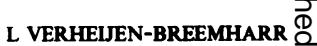
JM BOGAARD $\cong$ B VAN DEN BERG C HILVERING $\overrightarrow{0}$

Pathophysiology Laboratory $\overrightarrow{\vec{H}}$ Academisch Ziehenhuis $\omega$ 3015 GD Rotterdam The Netherlands $\times$

\section{Book notices}

AIDS Therapeutics in HIV Disease. M Youle, $J$ Clarbour, $\rightarrow$ P Wade, C Farthing. (Pp 162; £7.95.) Edinburgh: Churchill Livingstone, 1988. ISBN 0-443-04029-X.

This short pocket book, written largely by authors from St: Stephen's pharmacy and genitourinary medicine depart- ments, takes a systems orientated approach to the treatment $\overrightarrow{0}$ of problems related to human immunodeficiency virus (HIV) $\infty$ infection. There are 14 brief chapters (115 pages) covering fife main systems, plus chapters on HIV testing, retrovifiolo treatment, disinfection, psychological aspects, and termil care. The rest of the book comprises appendices-largetyo reproduced drug information sheets. The authors confesso that their book is written from their experience with homosexual men and it offers little specific guidance to the special areas of haemophilia, intravenous drug abuse, or paediatrics. Nevertheless the authors have valuable persona experience to relay at a time when respiratory physicians are increasingly seeing patients with pulmonary and other manifestations of AIDS. There are no illustrations other than a few line diagrams and tables. Certain areas of text could, $f$ think, have been tabulated to aid rapid reference by busyx clinicians. The large amount of cross referencing from one chapter to another was particularly irritating. It is unlikely? that a physician's diagnostic skills will be increased by this book as it is not intended to be a guide to treatment. The basic structure of the book would be improved if the clinicab systems review were consolidated into a chapter, the othe? chapters dealing with each infecting organism in turn. This would mean that, for instance, the diagnosis, clinical aspects? and treatment of cytomegalovirus infection were not deals with in four separate chapters. In certain areas the book appears to have been hastily compiled and there are minor inaccuracies and some omissions; doses of foscarnet, itra conazole, and ketoconazole are omitted, and the chapters org terminal care are vague and inadequate. An arrow is goins the wrong way in the flow chart on page 16 and the reason for the two separate prophylactic regimens is not clear. Nक mention is made of the suggestion from the United States that anti-tuberculosis treatment might need to be lifelong nor is there any real guidance on artificial ventilation or any mention of treatment for Legionella infection. Writter? 